that further ascents to greater heights will give a definite intensity of coincidence-producing rays near the top of the atmosphere.

A more detailed report will be published in the special number of the Zeitschrift für technische Physik for the Physikertagung, 1935.

We wish to thank the Deutsche Forschungsgemeinschaft for providing the means enabling us to make these investigations.

Erich Regener. Georg Pfotzer.

Physikalisches Institut,

Technische Hochschule, Stuttgart.

${ }^{1}$ E. Regener and G. Pfotzer, NATURe, 134, 325 ; 1934. Phys. Z., 35, $779 ; 1934$.

T. Thener. NaxuRe, 133, 364 ; 1933. Phys. Z., 34, 306; 1933. s B. Gross, Z. Phys., 83, 214 ; 1933.

\section{Radioactivity of Samarium}

SEvEral workers have investigated the radioactivity of samarium, and it is established that this element emits short-range $\alpha$-particles. Mäder ${ }^{1}$ has also reported the existence of a group of particles of range $1.37 \mathrm{~cm}$., which he believes to be protons. To obtain fuller information about these particles, I have introduced samarium sulphate into the emulsion of an Ilford ' $R$ ' plate, and examined the tracks which are produced. The technique of this method has been previously described ${ }^{2}$.

After seven weeks exposure, numerous tracks are observed on the plate, most of which clearly belong to the short-range $\alpha$-particle group. The accuracy with which the range in air can be determined from measurements of the length of the tracks is now known, and an account of the method used in making this determination will shortly be published. The range so determined is

$$
R=1 \cdot 13 \pm 0.02 \mathrm{~cm} \text {. standard air. }
$$

This value is in good agreement with those previously published.

In addition to these short tracks, we have observed tracks which correspond to particles of much longer range, up to at least $3 \cdot 5 \mathrm{~cm}$. air. These are much less numerous than the short tracks, the relative frequency being of the order $1: 100$. So far, some 150 such tracks have been measured. The distribution curve of the measurements does not show any welldefined maximum, such as is always found with a group of $\alpha$-particle tracks, and the 'scatter' of the measurements is much greater than is found, for example, with the tracks of uranium $\alpha$-particles, for which numerous measurements have been made. This alone is strong evidence that we are not dealing with a group of $\alpha$-particles. Furthermore, the mean separation of the grains is found to be $2 \cdot 0 \mu$ for the whole group, and many of the individual tracks show greater values. The mean separation for the short. range $\alpha$-particle tracks, obtained on the same plate, is $1 \cdot 6 \mu$. It has been pointed out previously ${ }^{2}$ that this forms a criterion for distinguishing between $\alpha$ particle tracks and proton tracks. It appears, therefore, that the long tracks cannot be attributed to $\alpha$-particles, and we must conclude that samarium emits singly-charged particles.

It is not yet possible to make any accurate statement as to the range of these particles. The distribution curve suggests that more than one group may be present, but the greatest range must be in the neighbourhood of $3.5 \mathrm{~cm}$. air. It is hoped that further observations will make it possible to decide these points.

I am indebted to Prof. Hevesy for supplying a specially pure sample of samarium, and to $\mathrm{Mr}$. Dabholkar of this Department for help in the measurements.

Department of Physics,

H. J. TAYLOR.

Wilson College,

Bombay.

Sept. 13.

1 Mäder, Z. Phys., 88, 601 ; 1934.

2 Taylor, Proc. Roy. Soc., A, 150, 382 ; June 1935.

\section{Emission of Positrons from Radioactive Sources}

WITH the improved apparatus, already used by us in the investigation of positrons from thoriumactive deposit, we have repeated our measurements of the positron spectrum, emitted by a thin-walled radon tube ; a new determination of the ratio of the positron number to that of the $\beta$-particles of $\mathrm{RaC}$ was also made. The positron spectrum is shown in Fig. 1: for each point about 1,000 particles were counted. The ratio $N_{+} / N_{\beta}$ was found to be $2-3 \times 10^{-4}\left(N_{+}=\right.$number of positrons, $N_{\beta}=$ number of $\beta$-particles of $\mathrm{RaC}$ ). Surrounding the source with a $1 \mathrm{~mm}$. lead sheet, we could compare the number of positrons emitted by the source with that produced by the $\gamma$-rays of $\mathrm{RaC}$ in lead: it was found that $N+(N+(\mathrm{Pb})=0.9-1$.

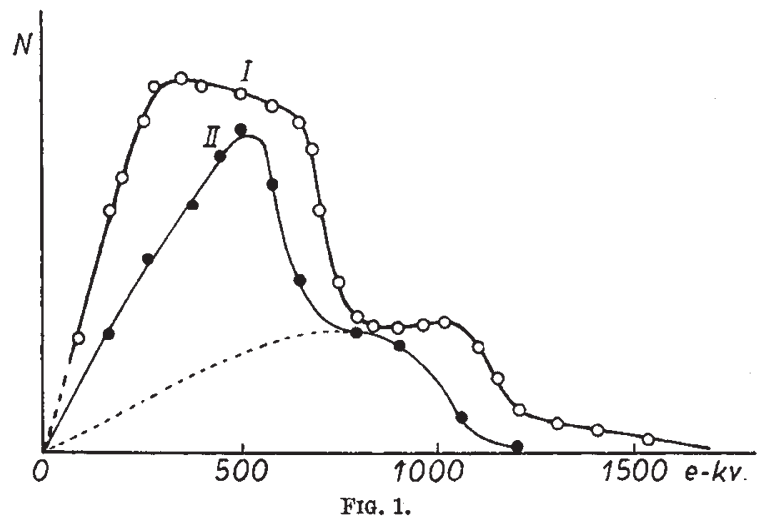

In the positron spectrum from the source, two dis. continuities are observed at 760 and l,200 e.kv., which correspond to the internal conversion of two strong $\gamma$-rays of $\mathrm{RaC}$ with energies 1,760 and 2,220 e.kv. The general shape of the spectrum does not correspond, however, either to the theoretical spectrum of internal conversion of $\gamma$-rays from $\mathrm{RaC}$, or to the positron spectrum, produced in the thin $(25 \mu)$ lead foil by $\gamma$-rays (curve II). By a method similar to that used in ouranalys is of the positron spectrum from thorium ${ }^{1}$, it is possible to separate the portion of the spectrum due to the internal conversion of $\gamma$-rays from the total spectrum. The remaining main portion of the spectrum has the features of a continuous spectrum extending in energy up to $1,700 \mathrm{e.kv}$. The number of positrons belonging to this portion of the spectrum is of the same order of magnitude as that in the spectrum of 